\title{
Response to a Letter to the Editor from Rodgers regarding "Overexpression of Myostatin2 in zebrafish reduces the expression of dystrophin associated protein complex (DAPC) which leads to muscle dystrophy"
}

\author{
Aseervatham Anusha Amali • Cliff Ji-Fan Lin · Yi-Hsuan Chen • \\ Wei-Lun Wang · Hong-Yi Gong · Ravikumar Deepa Rekha • \\ Jenn-Khan Lu $\cdot$ Thomas T. Chen · Jen-Leih Wu
}

Published online: 1 November 2008

(C) National Science Council Taipei 2008

We appreciate being made aware of earlier published work on the cloning of zebrafish myostatin 2 (MSTN-2), nomenclature of MSTN family. In the published version of this article, we reported one zebrafish MSTN-2 cDNA (GenBank accession number: AY614000), which is different from two published zebrafish MSTN-2 according to comparison of nucleotide and deduced amino acid sequences (Fig. 1b). However, two previous published articles which identified zebrafish MSTN-2 cDNA (GenBank accession number: AY687474) [1] and gene (GenBank accession number: AY693972) [2] was not referenced in this article. We apologize for neglecting pertinent references. According to new nomenclature of myostatin family [3], we construct new phylogenetic tree including three zebrafish MSTN-2 to replace old Fig. 3.

\section{References}

1. Kerr T, Roalson EH, Rodgers BD (2005) Phylogenetic analysis of the myostatin gene sub-family and the differential expression of a novel member in zebrafish. Evol Dev 7(5):390-400

2. Biga PR, Roberts SB, Iliev DB, McCauley LA, Moon JS, Collodi $\mathrm{P}$, Goetz FW (2005) The isolation, characterization, and expression of a novel GDF11 gene and a second myostatin form in zebrafish, Danio rerio. Comp Biochem Physiol B Biochem Mol Biol 141(2):218-230

3. Rodgers BD, Roalson EH, Weber GM, Roberts SB, Goetz FW (2007) A proposed nomenclature consensus for the myostatin gene family. Am J Physiol Endocrinol Metab 292(2):E371-E372

A. A. Amali - C. J.-F. Lin - Y.-H. Chen - W.-L. Wang ·

H.-Y. Gong · R. D. Rekha · J.-L. Wu ( $\varangle)$

Laboratory of Marine Molecular Biology and Biotechnology,

Institute of Zoology, Academia Sinica, Taipei, Taiwan

e-mail: jlwu@gate.sinica.edu.tw

\section{J.-F. Lin}

Graduate Institute of Life Sciences, National Defense Medical Center, Taipei, Taiwan

\section{W.-L. Wang}

Institute of Fisheries Sciences, National Taiwan University,

Taipei, Taiwan

J.-K. Lu

Department of Aquaculture, National Taiwan Ocean University, Keelung, Taiwan

\section{T. T. Chen}

Department of Molecular and Cell Biology and Biotechnology Center, University of Connecticut, Storrs, CT, USA 
Fig. 1b Alignment of deduced amino acids of three zebrafish MSTN-2, including MSTN-2c (AY614000), MSTN-2b (AY693972) and MSTN-2a (AY687474). Variant amino acid residues in $\mathrm{N}$-terminal of MSTN-2c and in unique domain within matured peptide of zebrafish MSTN-2c and MSTN$2 \mathrm{~b}$ are indicated with asterisks and underlined, respectively

\section{B}

ZfMSTN $-2 c$

z MSTN-2a

2fMSTN $-2 \mathrm{C}$ 2 FMSTN $-2 b$ : zFMSTN-2a:

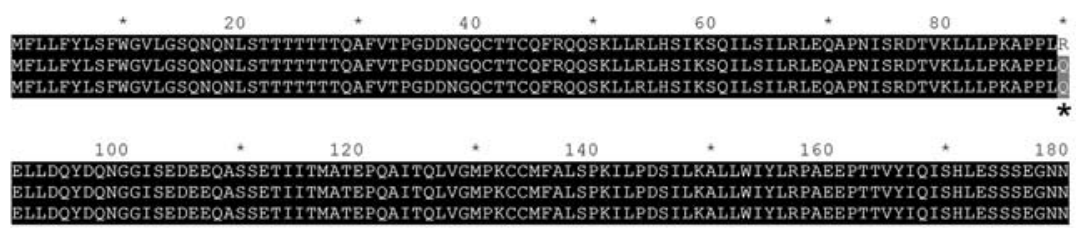

180 ZFMSTN-2a:

z $\mathrm{FMSTN}-2 \mathrm{c}$

f FMSTN-2b :

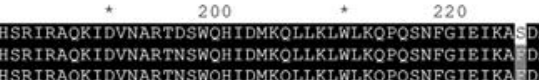

ANGNDLAV?

$240 \quad * \quad 260$

z FMSTN-2a : HSRIRAQKIDVNARTNSWQHIDMRQLLKLWLKQPQSNEGIEIKA

ANGNDLAVTS 法

\section{0}

300 CVRYPHSHIVNKA

*

2 IMSTN $-2 c$ zfMSTN- $2 b:$
z $\mathrm{FMSTN}-2 \mathrm{a}:$

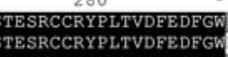
1 APKRYKANYC - 340

340

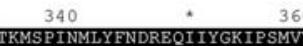
IYTKK IPSMVV : 360 TKMSPINMLY FNDREQI IYGKI I SMVY : 360 TKMSPINMLY FNDREOI I YGK I PSMVV : 360

ZfMSTN-2C:

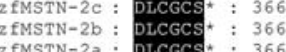

GCS * : 366 
Fig. 3 Phylogenetic tree of MSTNs. Phylogenetic and molecular evolutionary analyses were conducted using

Molecular Evolutionary Genetics Analysis MEGA 4 software (http://www. megasoftware.net/index.html). Tree construction and distance correction were followed by neighbor-joining and Poisson correction methods, respectively. Four GDF11 from mammalian and zebrafish were used as an out group. Sequences, in addition to those in Fig. 2, used in construction of this phylogenetic tree are baboon MSTN (AF019619), goose MSTN (AY448009), turkey MSTN (AF019625), chicken MSTN (AY448007), dog MSTN (AY367768), pig MSTN (AY448008), horse MSTN (AB033541), goat MSTN (AY436347), sheep MSTN (AF019622), blue catfish MSTN-1 (AY540992), channel catfish MSTN-1 (AF396747), gilthead seabream MSTN-1 (AF258448), gilthead seabream MSTN-2 (AY046314), striped bass MSTN-1 (AF290910), white perch MSTN-1

(AF290911), white bass MSTN1 (AF197194), shidrum MSTN1 (AF316881), shidrum MSTN2 (AY059386), human GDF-11 (NM_005811), mouse GDF-11 (NM_010272), rat GDF-11 (XM_343148) and zebrafish GDF-11 (AF411599)

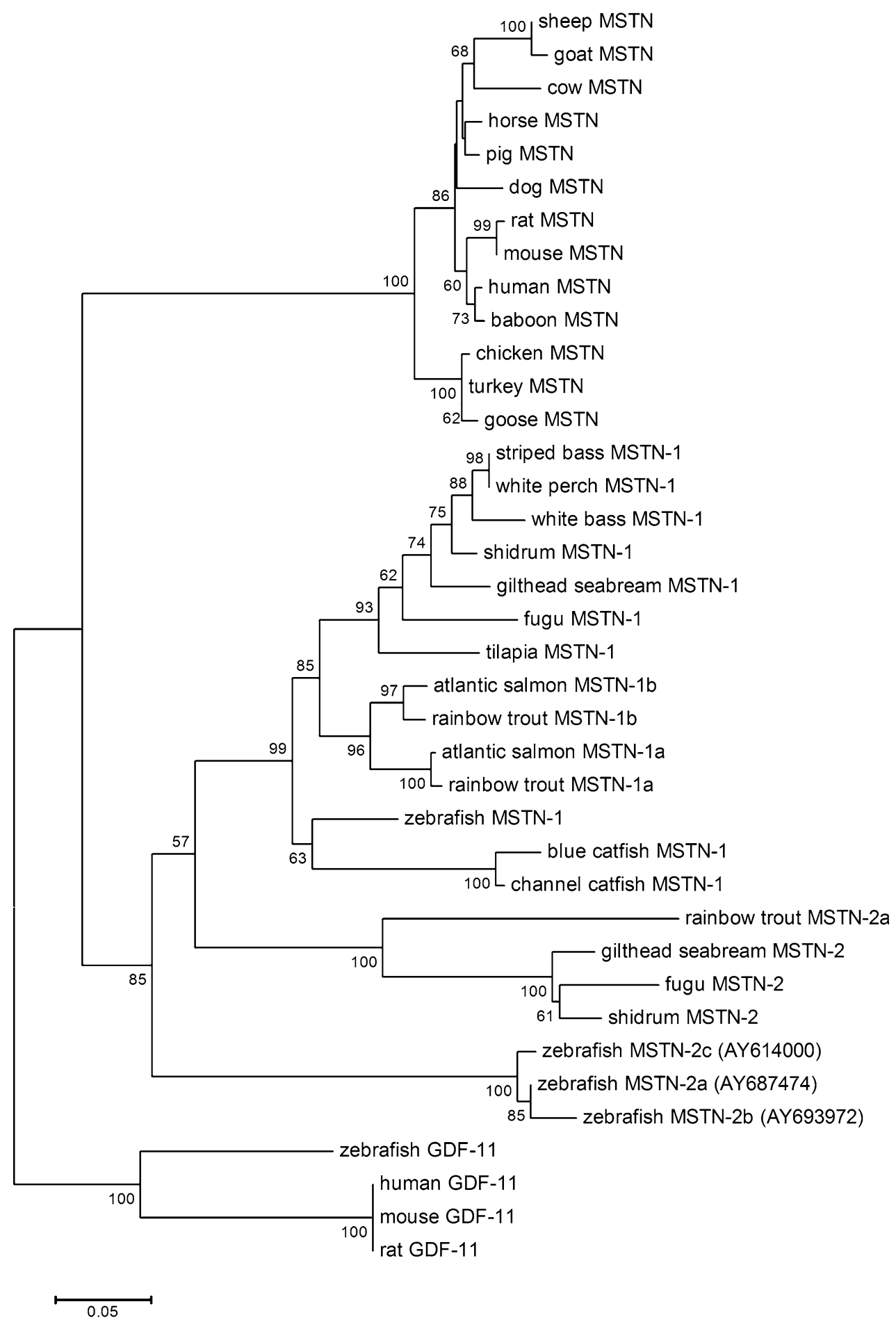

